\title{
miR-126 regulates the progression of epithelial ovarian cancer in vitro and in vivo by targeting VEGF-A
}

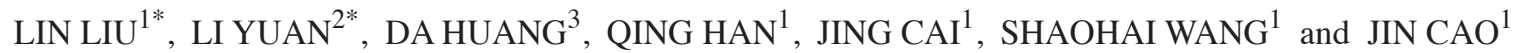 \\ ${ }^{1}$ Department of Obstetrics and Gynecology, Union Hospital, Tongji Medical College, Huazhong University of Science and \\ Technology, Wuhan, Hubei 430022; ${ }^{2}$ Department of Obstetrics and Gynecology, The First People's Hospital of Shangqiu, \\ Shangqiu, Henan 476100; ${ }^{3}$ Department of Clinical Laboratory, Union Hospital, Tongji Medical College, \\ Huazhong University of Science and Technology, Wuhan, Hubei 430022, P.R. China
}

Received August 4, 2019; Accepted May 19, 2020

DOI: $10.3892 /$ ijo.2020.5082

\begin{abstract}
MicroRNA (miRNA/miR-126) has been shown to be associated with ovarian cancer in previous studies. In ovarian cancer, however, the specific status of miR-126 remains largely unknown. In the present study, to clarify its role in ovarian cancer, the levels of miR-126 were first examined using laser microdissection and RT-qPCR. It was found that the miR-126 level was decreased in ovarian tissue samples and the restoration of miR-126 inhibited cell proliferation, cell invasion and migration in vitro and suppressed tumor growth in vivo. A bioinformatics search revealed that the angiogenesis-related gene, vascular endothelial growth factor (VEGF)-A, was among the potential targets of miR-126. The suppression of invasion and proliferation induced by ectopic miR-126 expression was nullified by the ectopic expression of VEGF-A, suggesting that these suppressive effects were largely attributable to the ability of miR-126 to target VEGF-A. Moreover, the restoration of miR-126 suppressed the angiogenic potential of human umbilical vein endothelial cells (HUVECs). On the whole, these findings indicate that the loss of expression of miR-126 contributes to the abnormal VEGF-A accumulation and subsequent unchecked cell invasion and cell proliferation in epithelial ovarian cancer.
\end{abstract}

\section{Introduction}

Even though the treatment strategies against epithelial ovarian cancer have witnessed marked improvements, epithelial

Correspondence to: Dr Shaohai Wang or Dr Jin Cao, Department of Obstetrics and Gynecology, Union Hospital, Tongji Medical College, Huazhong University of Science and Technology, 1277 Jiefang Avenue, Wuhan, Hubei 430022, P.R. China

E-mail: wangshaohai@medmail.com.cn

E-mail: jin_cao_union@163.com

*Contributed equally

Key words: ovarian cancer, miR-126, vascular endothelial growth factor-A, cell behavior, invasion, proliferation ovarian cancer remains the leading cause of cancer-related mortality from gynecological cancers (1). As the symptoms are vague and non-specific, these tumors often present at an advanced stage; in addition, the molecular mechanisms that govern its dissemination are not yet fully understood.

MicroRNA (miRNA/miR-126) is an intragenic miRNA. It is located on human chromosome 9, within the 7th intron of EGFL7 (2). The function of miR-126 in physiological and pathological processes has been extensively studied (3-5). miR-126 is found in endothelial cells and in highly vascularized tissues (6). In vivo studies have demonstrated miR-126 mutant mice develop leaky blood vessels, indicating that miR-126 plays an essential role in developmental angiogenesis and vascular integrity (7). In cancers, the downregulation of miR-126 is a frequent occurrence. The aberrant expression of miR-126 is closely related to a variety of cancers, such as hepatocellular carcinoma, breast cancer, thyroid cancer, gastrointestinal cancer and lung cancer $(2,8)$. Research has indicated (5) that miR-126 can inhibit cell proliferation, invasion and migration by targeting solute carrier family 7 member 5 (SLC7A5), SRY-box transcription factor 2 (SOX-2), insulin receptor substrate 1 (IRS-1), homeobox A9 (HOXA9), ADAM metallopeptidase domain 9 (ADAM9), CRK proto-oncogene, adaptor protein (CRK), KRAS, epidermal growth factor (EGF)-like domain multiple 7 (EGFL7), phosphoinositide 3-kinase (PI3K) and vascular endothelial growth factor (VEGF).

The association between miR-126 and epithelial ovarian cancer has been reported in previous studies $(9,10)$. However, limited research has been conducted on its expression and biological function in ovarian cancer (11-13). In the present study, laser microdissection was used to ensure specimen homogeneity. The expression of miR-126 was examined using clinical specimens and in vivo animal and in vitro cell models. The interaction between miR-126 and VEGF-A and its role in epithelial ovarian cancer were investigated.

\section{Materials and methods}

Tissue samples. Tumor and normal specimens were obtained from the Gynecologic Tissue Bank of Wuhan Union Hospital with written informed consent and ethical approval (February, 
2004 to June, 2016). The present study was approved by the Ethics Committee of Union Hospital, Tongji Medical College, Huazhong University of Science and Technology, China. In total, 10 normal ovarian tissues (germinal epithelium from patients with adenomyosis or myoma) and 36 malignant epithelial tumors ( 1 clear cell tumor, 3 endometrioid carcinomas, 6 mucinous cystadenocarcinomas and 26 serous cystadenocarcinomas) were analyzed. No patients had been subjected to previous chemotherapy. All diagnoses were pathologically confirmed.

Laser microdissection (LMD). To ensure the homogeneity of the specimens, specimens with $<90 \%$ cancerous content were subjected to LMD. LMD was performed according to the method described in the study by Cai et al (14). Briefly, serial sections (8- $\mu \mathrm{m}$-thick) and a $4-\mu \mathrm{m}$-thick section were cut from formalin-fixed paraffin-embedded (FFPE) samples for LMD and $\mathrm{H} \& \mathrm{E}$ staining, respectively. Immediately prior to LMD, the sections were deparaffinized, stained with $1 \%$ cresyl violet (Sigma-Aldrich; Merck KGaA) for $1 \mathrm{~min}$, dehydrated through 75, 95 and $100 \%$ alcohol grades for $30 \mathrm{sec}$ each, and finally immersed in xylene for $3 \mathrm{~min}$ and air-dried for $1 \mathrm{~min}$. The sections were deparaffinized and dehydrated prior to microdissection, which was carried out using an ArcturusXT LCM instrument (Applied Biosystems-Life Technologies; Thermo Fisher Scientific, Inc.). The AutoScan ${ }^{\mathrm{TM}}$ XT1.2 analysis software module was used for visualization. The captured cells (approximately 5,000 per specimen) were used in subsequent analysis.

Cell lines and culture. SKOV3 and ES2 cell lines were purchased from the China Center for Type Culture Collection. The former was derived from adenocarcinoma, and the latter was derived from ovarian clear cell carcinoma. These two cell lines were propagated in Dulbecco's modified Eagle's medium (Gibco; Thermo Fisher Scientific, Inc.) supplemented with $10 \%$ fetal bovine serum (Gibco; Thermo Fisher Scientific, Inc.). Cell lines were cultured at $37^{\circ} \mathrm{C}$ in a humidified incubator with $5 \% \mathrm{CO}_{2}$.

Isolation and culture of HUVECs. Human umbilical cords were collected with written informed consent and ethical approval provided the the Ethics Committee of Union Hospital, Tongji Medical College, Huazhong University of Science and Technology, Wuhan, China. Human umbilical cords were collected from 24 pregnant women (38-41 weeks of gestational age, abdominal delivery, singleton, with no complications) at Wuhan Union Hospital (from March, 2014 to September, 2014). Endothelial cells (ECs) were isolated from the umbilical vein vascular wall and cultured as previously described by Xie et al (15). HUVECs were identified with two endothelial cell markers, factor VIII related antigen [von Willebrand factor (vWF)] and CD31 [platelet endothelial cell adhesion molecule-1 (PECAM-1)]. Prior to identification, the isolated cells were fixed with $4 \%$ paraformaldehyde and sealed with $1 \%$ bovine serum albumin (Gibco; Thermo Fisher Scientific, Inc.) for $30 \mathrm{~min}$ at room temperature sequentially. For immunofluorescence identification, the isolated cells were treated with primary antibody to Factor VIII related antigen (1:100, cat. no. PB0086, Boster Biological Technology) and CD31 (1:500, cat. no. M01513; Boster Biological Technology) at $4^{\circ} \mathrm{C}$ in a humid chamber overnight. Subsequently, the cells were washed with phosphate-buffered saline (PBS) 3 times at room temperature, and then incubated with biotinylated secondary antibody from SABC-Cy3 kit (1:100; cat. no. SA1078; Boster Biological Technology) for $30 \mathrm{~min}$ at at $37^{\circ} \mathrm{C}$. Before the cells were counterstained with SABC-Cy3 from the SABC-Cy3 kit (1:100; cat. no. SA1078; Boster Biological Technology) in the dark for $30 \mathrm{~min}$ at $37^{\circ} \mathrm{C}$, they were washed with PBS 3 times at room temperature. After washing with PBS 5 min for 4 times at room temperature, the cells were visualized under a fluorescence microscope (IX71; Olympus Corporation).

Transfection of RNA oligoribonucleotides. Transfection was performed using Lipofectamine 2000 reagent (Invitrogen; Thermo Fisher Scientific, Inc.) following the manufacturer's protocol. At 1 day prior to transfection, cells were plated in 6 -well plates $\left(2 \times 10^{5} /\right.$ well). The following day, or when the cells were $70 \%$ confluent, they were transfected with either 100 pmol of miR-126 mimics (target sequence, 5'-UCGUACCGUGAGUAAUAAUGCG-3' and 5'-CAU UAUUACUCACGGUACGAUU-3'; GenePharma), miR-126 inhibitors (target sequence, 5'-CGCAUUAUU ACUCACGGUACGA-3'; GenePharma) or the negative control siRNAs (MNC and INC) (GenePharma). Subsequent experiments were performed at 24-48 h following transfection.

Lentiviruses packaging and stable cell lines. Lentiviral constructs with hsa-miR-126 or a hsa-miR-scrambled control vectors were constructed, packaged and validated by GenePharma. For LV-miR-126 transfection, SKOV3 and ES2 cells were seeded into 6 -well plates at $3.5 \times 10^{5}$ cells/well. Following propagation for $24 \mathrm{~h}$, viral particles $\left(3 \times 10^{7}\right)$ were added.

Recombinant human VEGF. Recombinant human VEGF (cat. no. 100-20; PeproTech) was added at a concentration of $40 \mathrm{ng} / \mathrm{ml}$ to lentivirus-miR-126 (LV-miR-126)-infected cells. Following incubation for $24 \mathrm{~h}$ at $37^{\circ} \mathrm{C}$, RNA and protein were collected for RT-qPCR and western blot analysis, respectively as described below. The infected cells were used for migration, invasion and cell proliferation experiments.

Bioinformatics analyses. For miRNA target gene prediction, TargetScan online software (http://www.targetscan.org/), miRanda (http://www.microrna.org/microrna/getDownloads. do) and PicTar (http://pictar.mdc-berlin.de/) were used.

$R N A$ extraction and $R T-q P C R$. Total RNA was extracted from the FFPE specimens and cultured cell using the RNeasy FFPE kit (Qiagen) and TRIzol reagent (Invitrogen; Thermo Fisher Scientific, Inc.), respectively. cDNA was synthesized using the RevertAid ${ }^{\mathrm{TM}}$ First Strand cDNA Synthesis kit (MBI Fermentas) according to the supplied protocol. miR-126 was amplified in triplicate using a Hairpin-it ${ }^{\mathrm{TM}}$ miRNAs qPCR Quantitation kit (GenePharma) on a StepOnePlus system (Applied Biosystems; Thermo Fisher Scientific, Inc.) following the manufacturer's instructions. The levels of miR-126 expression were normalized to U6. The cycling parameters for the reverse transcription reaction were $16^{\circ} \mathrm{C}$ for $30 \mathrm{~min}, 42^{\circ} \mathrm{C}$ for $30 \mathrm{~min}, 85^{\circ} \mathrm{C}$ for $10 \mathrm{~min}$ and a hold at $4^{\circ} \mathrm{C}$. The amplification 
conditions were as follows: Initial 1 step at $95^{\circ} \mathrm{C}$ for $3 \mathrm{~min}$, followed by 40 cycles at $95^{\circ} \mathrm{C}$ for $12 \mathrm{sec}$ and with a final extension step at $62^{\circ} \mathrm{C}$ for $60 \mathrm{sec}$. The primers used were as follows: VEGF-A forward, 5'-GAACTTTCTGCTGTCTTGG-3' and reverse, 5'-TTTTCTTGTCTTGCTCTATCT-3'; and $\beta$-actin forward, 5'-GCCAACACAGTGCTGTCTGG-3' and reverse, 5'-GCTCAGGAGGAGCAATGATCTTG-3'. The expression level of each gene was calculated using the $2^{-\Delta \Delta \mathrm{Cq}}$ method (16).

Transwell assay. Cell migration and invasion were measured using Transwell ${ }^{\circledR}$ chambers ( $8 \mu \mathrm{m}$ pore size; Corning Inc.). For the Transwell assay, at $24 \mathrm{~h}$ following transfection with miR-126 mimics and miRNA-126 inhibitors, $1.0 \times 10^{5}$ cells (3 replicates per group) were suspended in serum-free medium and seeded into the upper chamber with $8 \mu \mathrm{m}$ pore filters, and $600 \mu \mathrm{l}$ of DMEM with $10 \%$ FBS was added to the lower chamber. The cells were then incubated for $24 \mathrm{~h}$ at $37^{\circ} \mathrm{C}$. Migrated cells were stained with crystal violet (Beyotime Institute of Biotechnology) for $20 \mathrm{~min}$ at room temperature and observed under an optical microscope (IX71; Olympus Corporation). For the invasion assay, the protocols were similar to those for the Transwell migration assays, with the difference being that an aliquot of Matrigel (50 $\mu \mathrm{l})$ (Corning Inc.) was applied to the upper surface to mimic the basement membrane. The results were analyzed using Image-Pro Plus, v6.0 (Media Cyberbetics).

Wound-healing assays. Following $12 \mathrm{~h}$ of transfection with miR-126 mimics and miR-126 inhibitors, the SKOV3 cells were plated at $1 \times 10^{6}$ per well into 6 -well plates and allowed to reach $90 \%$ confluence. The monolayer was scratched using a $200 \mathrm{ml}$ pipette tip after the cells were serum-starved for $12 \mathrm{~h}$ and washed with serum-free medium to remove detached cells. The cells were cultured with serum-free medium. At 0 and $24 \mathrm{~h}$, an inverted microscope (IX71; Olympus Corporation) was utilized to visualize the wound healing and obtain images. The percentage migration was calculated using the following equation: $[\Delta$ area/area (day 0$)] \times 100$.

Cell proliferation assay. Cell proliferation was evaluated using the Clik-iT ${ }^{\circledR}$ EdU Imaging kit and Click-iT ${ }^{\circledR}$ reaction cocktail (Invitrogen; Thermo Fisher Scientific, Inc.) according to the manufacturer's protocol. The results were analyzed using Image-Pro Plus, v6.0 (Media Cyberbetics). Cell viability was assessed using trypan blue staining (Invitrogen; Thermo Fisher Scientific, Inc.). Following transfection, the cells were stained by $0.4 \%$ trypan blue for $3 \mathrm{~min}$ at room temperature. The growth rate was determined by trypsinization and counting the number of viable cells (trypan blue exclusion) on a hematocytometer in triplicate every $12 \mathrm{~h}$ for 3 days.

F-actin immunofluorescence. Following $48 \mathrm{~h}$ of transfection with miR-126 mimics and miR-126 inhibitors, the SKOV3 cells were cultured at $5 \times 10^{5}$ per well in 12 -well plates and allowed to adhere for $24 \mathrm{~h}$. SKOV3 cells were grown to $75 \%$ confluency on uncoated glass cover slips in a 12-well plate. The cells were fixed in $3.7 \%$ paraformaldehyde for $10 \mathrm{mins}$ followed $0.1 \%$ Triton $\mathrm{X}-100$ for $15 \mathrm{~min}$ at room temperature. The cells were maintained for $60 \mathrm{~min}$ at room temperature in rhodamine-phalloidin solution. Finally, nuclei were stained with
Hoechst 33342 (Invitrogen Life Technologies; Thermo Fisher Scientific, Inc.) for $30 \mathrm{~min}$ at room temperature. Images were captured using an IX71 digital camera (Olympus Corporation).

Tube formation assay. Tube formation assay was used to evaluate the effects of miR-126 on HUVECs according to a previously published study with a few modifications (17). Briefly, the HUVECs were seeded in a Matrigel-coated plate at a density of $5 \times 10^{4}$ cells per well. Following $1 \mathrm{~h}$ of incubation at $37^{\circ} \mathrm{C}$, the HUVECs were incubated with LV-miR-126 supernatant for $24 \mathrm{~h}$ at $37^{\circ} \mathrm{C}$ and $5 \% \mathrm{CO}_{2}$. Tube formation was observed using an inverted microscope (Leica DMI6000B; Leica Microsystems $\mathrm{GmbH})$.

Enzyme-linked immunosorbent assay (ELISA) for VEGF. The levels of VEGF-A in conditioned media were measured using a human ELISA kit (cat. no. K5363-100; NeoBioscience) as per the manufacturer's instructions.

Protein extraction and western blot analysis. Cells were washed twice with cold PBS, then lysed using cell lysis (RIPA) buffer (Beyotime Institute of Biotechnology). Cell lyses were centrifuged at $13,000 \times \mathrm{g}$ for $10 \mathrm{~min}$, and the supernatants were collected and stored in aliquots at $-80^{\circ} \mathrm{C}$ after the protein concentration was measured using the bicinchoninic acid (BCA) assay. Total cell extracts were resolved on a $12 \%$ SDS polyacrylamide gels ( $25 \mu \mathrm{g}$ of protein were loaded per lane) and blotted onto a Hybond PVDF membrane (GE Healthcare Life Sciences). The membranes were blocked with blocking buffer [5\% non-fat dry milk in tris-buffered saline containing $0.1 \%(\mathrm{v} / \mathrm{v})$ Tween-20 (TBST)] for $1 \mathrm{~h}$ at room temperature. The membranes were then incubated overnight at $4^{\circ} \mathrm{C}$ with VEGF (1:2,000 dilution; cat. no. 500-M88; Peprotech), E-cadherin (1:500 dilution; cat. no. 3195; Cell Signaling Technology, Inc.), vimentin (1:500 dilution; cat. no. 5741; Cell Signaling Technology, Inc.), matrix metalloproteinase (MMP)2 (1:1,000 dilution; cat. no. 2763-1; Epitomics; Abcam) and $\beta$-actin (1:1,000 dilution; cat. no. sc-8432; Santa Cruz Biotechnology, Inc.). Following three 10-min washes in TBST, the membranes were incubated for $2 \mathrm{~h}$ at room temperature with a horseradish peroxidase-conjugated secondary antibody at 1:5,000 in blocking solution (cat. no. sc-2357; Santa Cruz Biotechnology, Inc.) and visualized using an enhanced chemiluminescence kit (Pierce; Thermo Fisher Scientific, Inc.).

Histological and immunohistochemical analysis. Immunohistochemical analyses were performed according to standard procedures (18). Antibodies and reagents for immunocytochemistry (IHC) included: Anti-VEGF-A (1:1,00 dilution; cat. no. BA0407; Wuhan Boster Biological Technology, Ltd.), anti-MMP2 (1:50 dilution; cat. no. 40994; Cell Signaling Technology, Inc.), anti-vimentin (1:100 dilution; cat. no. 5741; Cell Signaling Technology, Inc.), anti-E-cadherin (1:100 dilution; cat. no. 3195; Cell Signaling Technology, Inc.) and biotinylated goat anti-rabbit secondary antibody (1:100; cat. no. sc-2004; Santa Cruz Biotechnology, Inc.). The sections were incubated with the above-mentioned primary antibodies at $4^{\circ} \mathrm{C}$ overnight, followed by $1 \mathrm{~h}$ of incubation with the secondary antibody. Images were captured using an IX71 digital camera (Olympus Corporation). 
A

Normal
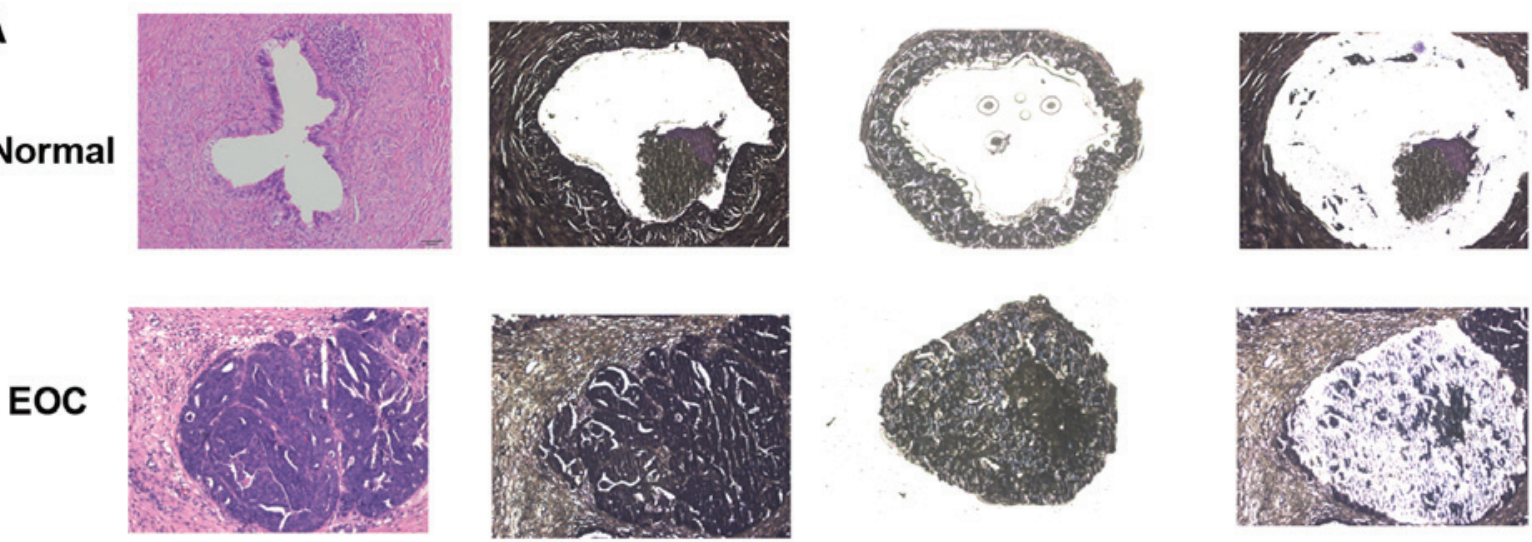

HE

Pre-microdissection Post-microdissection

Microdissected cells

B

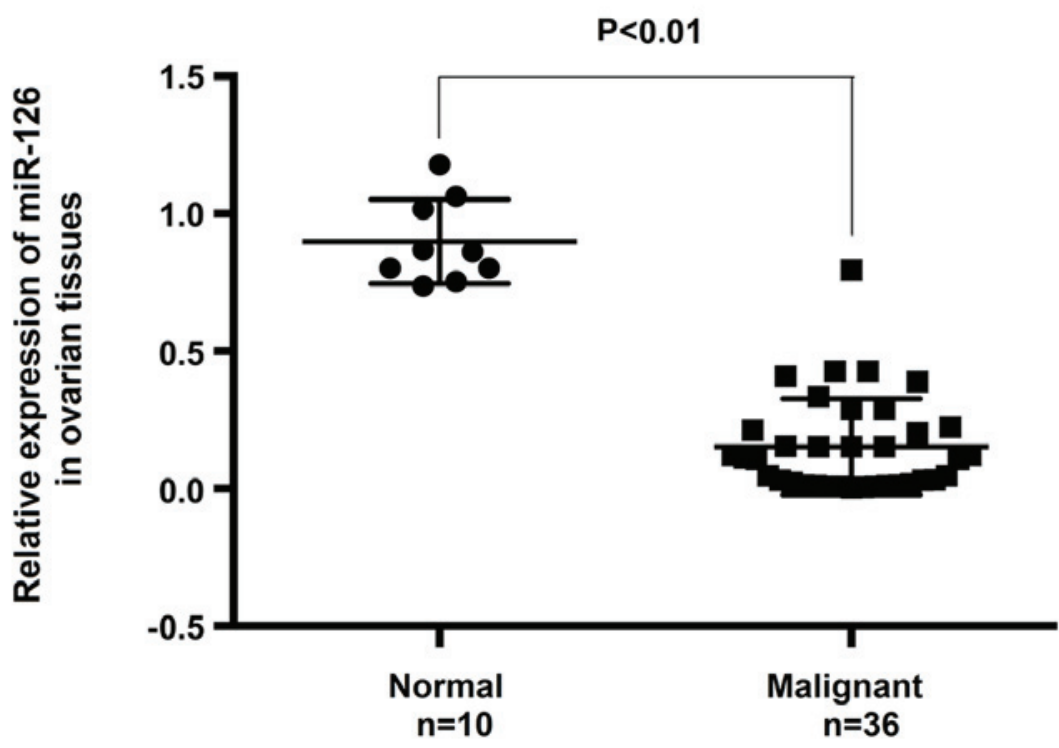

Figure 1. Differential expression of miR-126. (A) Laser microdissection (LMD) of normal tissues (H\&E, pre-mcrodissection, post-microdissection, microdissected cells, upper panel) and epithelial ovarian cancer (H\&E, pre-mcrodissection, post-microdissection, microdissected cells, lower panel) tissues from human ovarian tissues. (B) Expression levels of miR-126 in 10 normal ovarian tissues and 36 epithelial ovarian cancer tissues were examined by RT-qPCR. EOC, epithelial ovarian cancer.

Ovarian tumor xenograft model. Female BALB/c nude mice ( $\mathrm{n}=10$; age, 5 weeks; weight, $17-18 \mathrm{~g}$ ) were randomly separated into two groups, the LV-miR-126 and negative control (NC), ( $\mathrm{n}=5$ mice/group) and used for tumor formation assay. The housing conditions of the animals were as follows: Temperature, $23 \pm 1^{\circ} \mathrm{C}$; humidity, 40-70\%; 12-h dark/light cycle; and free access to food and water. LV-miR-126 SKOV3 cells or negative control cells $\left(5 \times 10^{6}\right.$ cells /mouse) were injected subcutaneously into the dorsal flanks of the nude mice. The tumor sizes were measured every 4 days using micrometer calipers. Tumor volumes were calculated according to the formula: $1 / 2 \times$ length $x$ width ${ }^{2}$. After 30 days, the mice were euthanized by cervical dislocation and the tumor tissues were harvested; death was confirmed by the completely termination of the heartbeat and breathing, as well as the disappearance of the foot withdrawal reflex. All animal studies were approved by the Institutional Animal Care and Use Committee at Tongji Medical College, Huazhong University of Science and Technology.
Statistical analysis. SPSS software v12.0 (SPSS Inc.) was used to perform statistical analysis. Data are expressed as the means \pm SD. Multigroup comparisons of the means were carried out by one-way analysis of variance (ANOVA) test with Tukey's post hoc test. $\mathrm{P}<0.05$ was considered to indicate a statistically significant difference. Spearman's correlation analysis was utilized to determine the correlation between miR-126 and VEGF-A expression.

\section{Results}

miR-126 expression is downregulated in epithelial ovarian cancer. Previous reports have associated miR-126 with epithelial ovarian cancer. In the present study, to clarify whether miR-126 is downregulated in EOC, the expression of miR-126 was determined in 46 tissue specimens by RT-qPCR. To ensure the homogeneity of the specimens, 21 specimens (out of 46 specimens) with $<90 \%$ cancerous content were subjected to LMD. The results revealed that the expression 
A

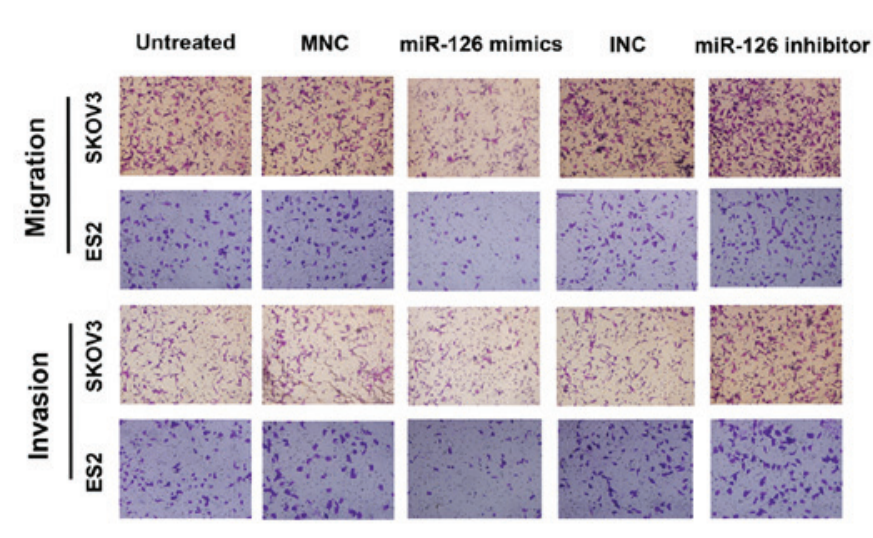

B

$\mathbf{O h}$
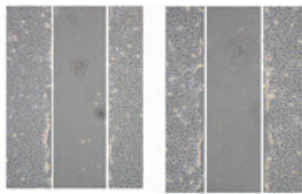

$24 \mathrm{~h}$

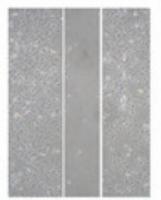

Untreated

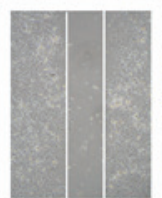

MNC
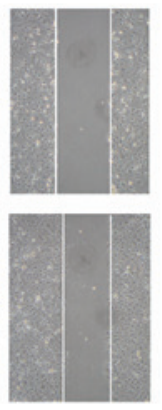

miR-126 mimics
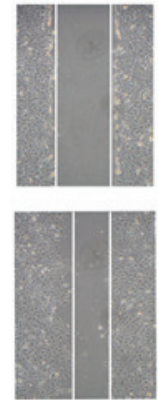

INC
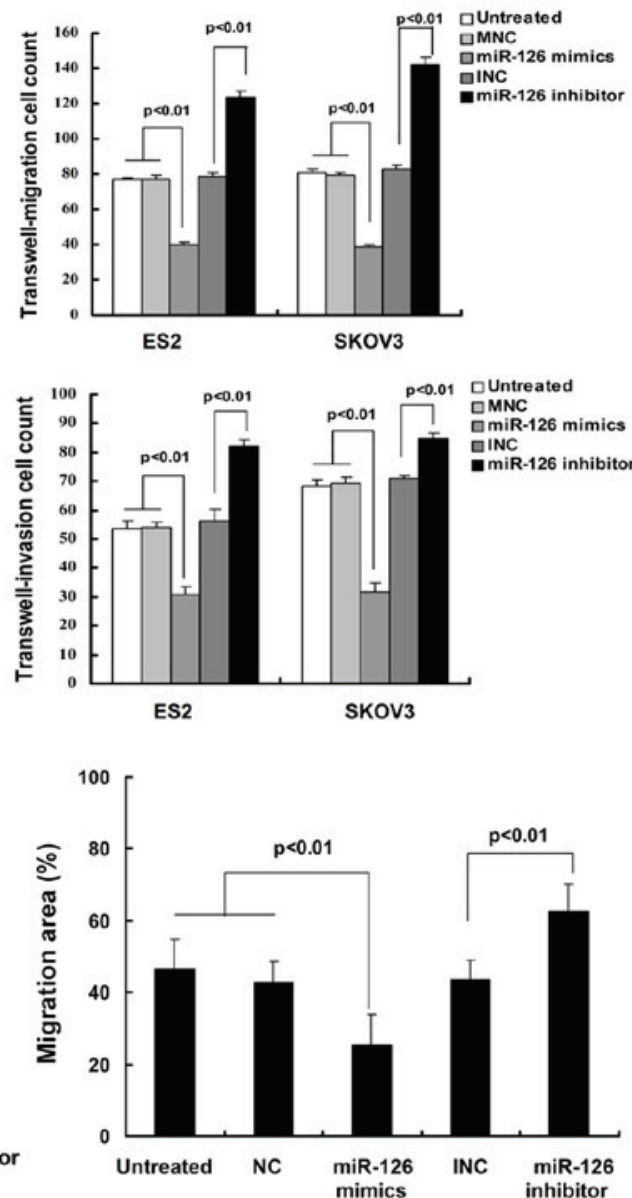

C
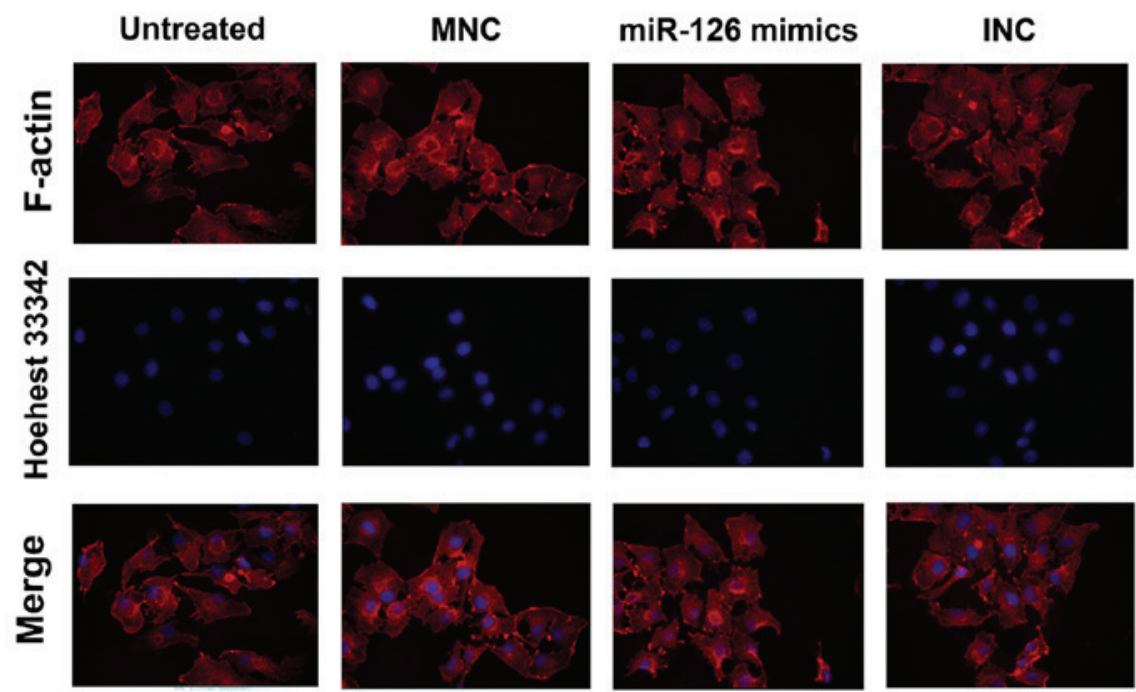

miR-126 inhibitor
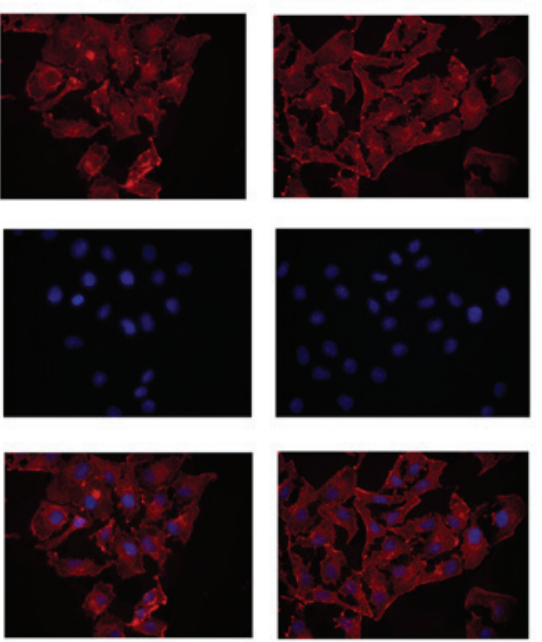

Figure 2. miR-126 inhibits ovarian cancer cells migration and invasion. (A) Transwell migration and invasion assay of SKOV3 and ES2 cells treated with miR-126 mimics or inhibitor. The cells were photographed at x200 magnification. (B) The migration of the indicated cells was detected by wound-healing assay. Magnification, x200. (C) F-actin was labeled by phalloidin in SKOV3 cells. The red signal represents the staining of F-actin, the blue signal represents the nuclear DNA staining by Hoechst 33342. Magnification, x400. MNC, mimic negative control; INC, inhibitor negative control.

of miR-126 was lower in malignant tumors than in normal ovarian epithelium (Fig. 1).

miR-126 suppresses cancer-relevant traits in vitro. To examine the effects of miR-126 on cell migration and proliferation, Transwell, wound-healing, EdU incorporation and trypan blue exclusion assays were used. The SKOV3 and ES2 cells were transfected with miR-126 mimics or inhibitors. The results indicated that the ectopic expression of miR-126 decreased the invasive and migratory ability of the SKOV3 and ES2 cells at $36 \mathrm{~h}$ following transfection (Fig. 2A). These observations were confirmed by a wound-healing assay (Fig. 2B). However, 
A
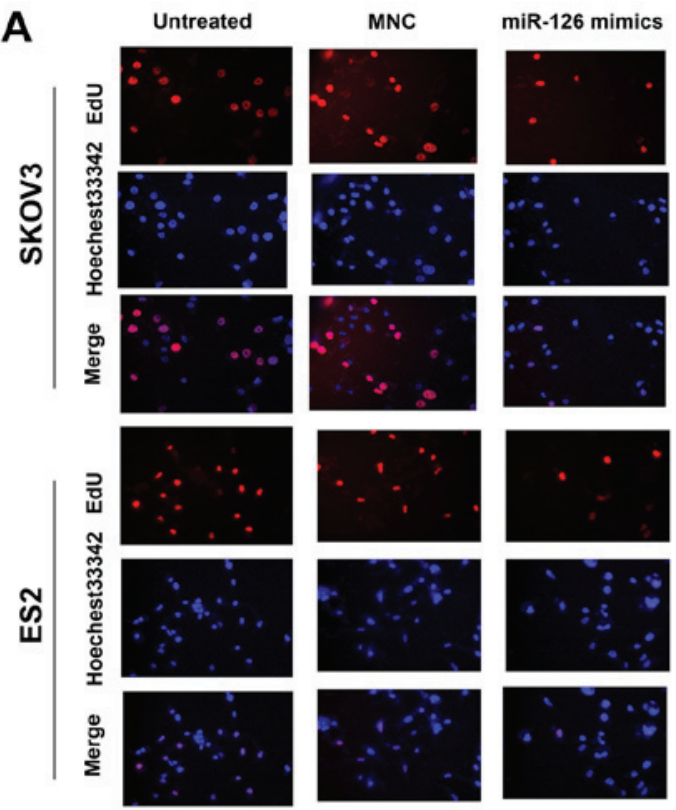

B

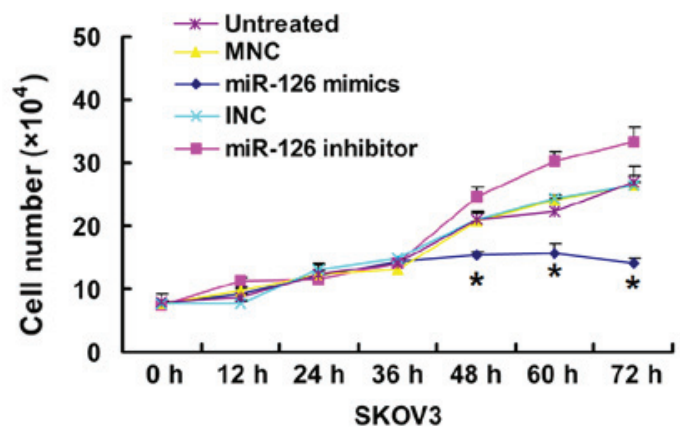

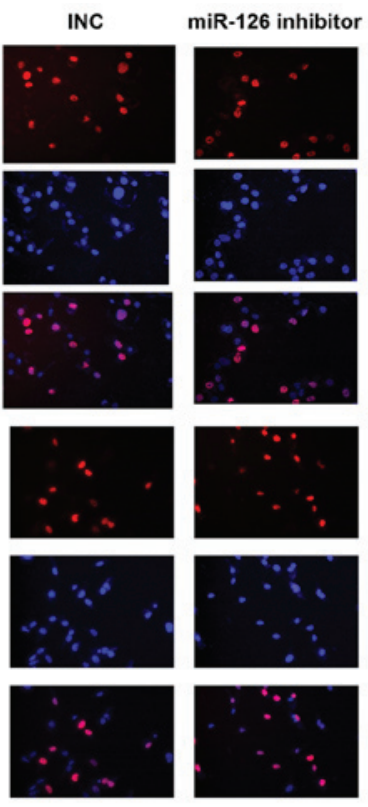
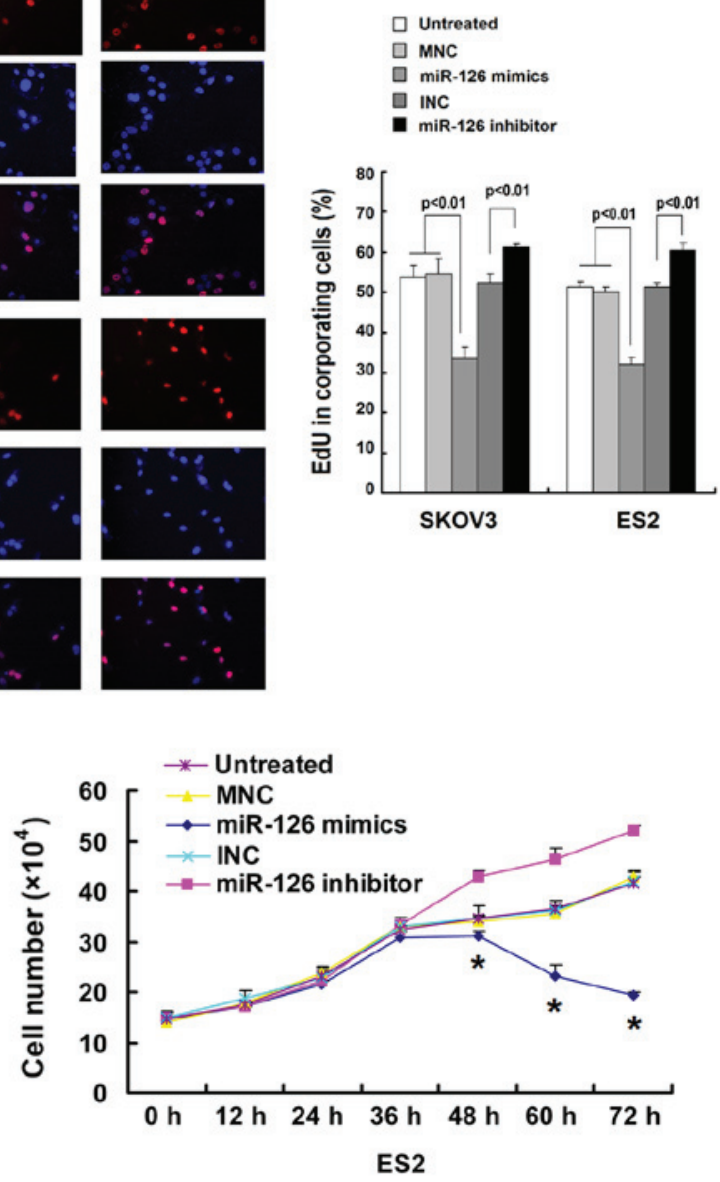

Figure 3. miR-126 suppresses cell proliferation in ovarian cancer. (A) The proliferation of SKOV3 and ES2 cells was determined by EdU assay. Magnification, $\mathrm{x} 400$. (B) Cell viability was measured by trypan blue dye exclusion test at 0, 12, 24, 36, 48, 60 and 72 h in SKOV3 and ES2 cells; "P<0.05 compared to MNC, INC and untreated cells. MNC, mimic negative control; INC, inhibitor negative control.

the inhibition of miR-126 reversed these effects. The ectopic expression of miR-126, however, did not markedly affect the cytoskeleton in vitro (Fig. 2C). It was also found that the overexpression of miR-126 decreased the cell proliferation rates, and the downregulation of miR-126 increased the cell proliferation rates at $48 \mathrm{~h}$ following transfection (Fig. 3A). These observations were confirmed by the trypan blue exclusion assay (Fig. 3B). The miR-126 mimic negative control (MNC) and the miR-126 inhibitor negative control (INC) failed to influence migration, invasion and proliferation.

miR-126 regulates the expression of $V E G F-A$. It has previously been reported that miR-126 targets VEGF-A in other tumors (5). In the present study, to determine whether this is the case in epithelial ovarian cancer, SKOV3 and ES2 cells were infected with lentivirus-miR-126 (LV-miR-126), and the expression levels of VEGF-A were determined by RT-qPCR and ELISA. As shown in Fig. 4A and B, the ectopic expression of miR-126 decreased the expression of VEGF-A in SKOV3 and ES2 cells at both the mRNA and protein level. Moreover, infection with LV-miR-126 downregulated the expression of the VEGF-A effector, MMP2, and upregulated the expression of the epithelial marker, E-cadherin, while no difference was observed between the negative control and untreated cells (Fig. 4C). Despite these findings, no reduction in the mesenchymal marker, vimentin, was observed when miR-126 expression was modulated (Fig. 4C). Furthermore, miR-126 expression negatively correlated with VEGF-A expression in epithelial ovarian cancer tissues $\left(\mathrm{R}^{2}=0.5711\right.$; Fig. 4D).

Ectopic VEGF-A expression reverses the miR-126-mediated effects on the invasion, proliferation defects and tube formation of HUVECs. To determine whether in vitro phenotypes associated with miR-126 expression can be reversed by the restoration of VEGF-A expression, recombinant human VEGF-A was added to LV-miR-126-infected SKOV3 and ES2 cells and the VEGF-A levels were assessed. In these cells, the ectopic expression of VEGF-A reversed the miR-126-imposed invasion and proliferation defects (Fig. 5A-C). HUVECs were identified morphologically and by immunofluorescence in terms of origin and purity. It was found that $>95 \%$ of the cells presented typical features of endothelial cells, such as a cobblestone pattern and the expression of Factor VIII related antigen and CD31 (Fig. 5D and E). Tube formation assays revealed that the HUVECs treated with LV-miR-126-infected supernatant formed fewer dendritic and tube-like structures than what was formed by the control cells. However, the number of these structures was increased on recombinant human VEGF spike (Fig. 5F). 
A

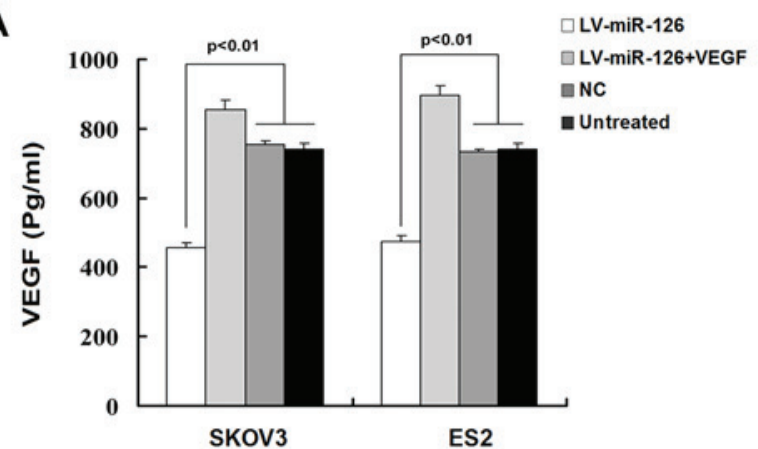

C

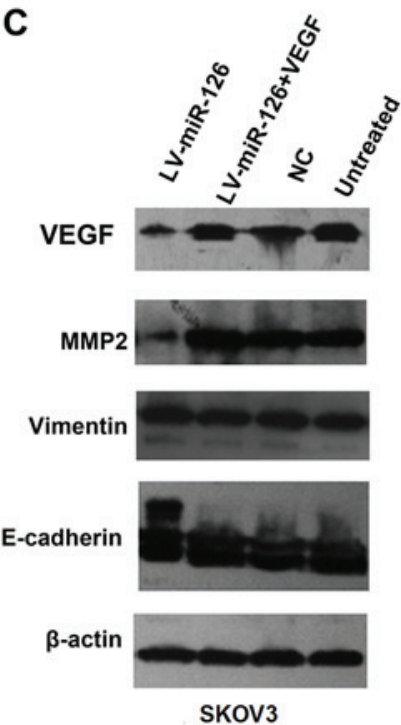

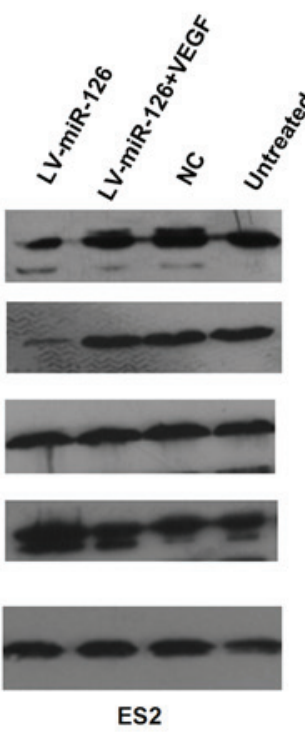

B

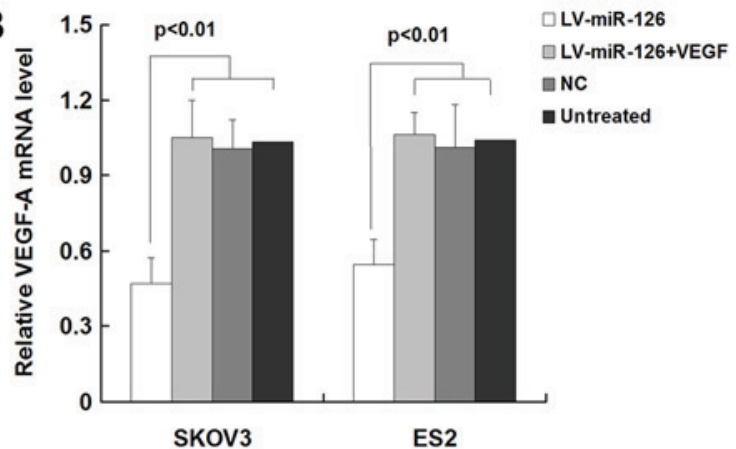

D

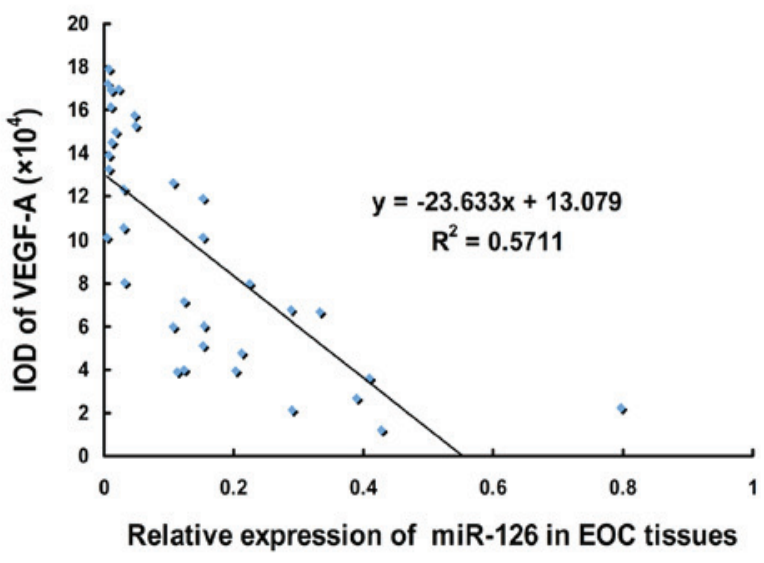

Figure 4. miR-126 inhibits cell metastasis and proliferation via VEGF-A by reducing MMP2 expression in EOC cells and EOC tissues. (A) The expression of VEGF-A in SKOV3 and ES2 cells were measured using an ELISA kit. (B) RT-qPCR for VEGF-A mRNA levels. (C) The protein levels of VEGF-A, MMP-2, vimentin and E-cadherin were determined by western blot analysis. (D) Expression of miR-126 and its correlation with VEGF-A in EOC tissues. NC, negative control.

miR-126 inhibits tumor growth in vivo. To investigate the role of miR-126 in vivo, LV-miR-126 SKOV3 cells and NC cells were injected subcutaneously into nude mice. The volume of subcutaneous tumors was measured every 4 days. The tumor volume was suppressed to a greater extent in the miR-126 overexpression group than in the NC group (Fig. 6A and B). Moreover, the results of RT-qPCR revealed that the VEGF-A mRNA level was much lower in the LV-miR-126 SKOV3 cells than in the NC cells (Fig. 6C). Immunohistochemistry was performed to investigate the levels of VEGF-A, MMP2, vimentin and E-cadherin in tumor xenografts. The higher expression of E-cadherin, and the lower expression of VEGF-A and MMP2 was found in the LV-miR-126 xenografts compared with the NC xenografts (Fig. 6D).

\section{Discussion}

In ovarian cancer, previous expression profiling of clinical tumors has provided contradictory findings as regards the status of miR-126. One study found it to be highly expressed (19), while it was found to be downregulated in another (20). The specific status of miR-126 in ovarian cancer has been far from conclusive as these profiling studies assessed neither tumor contents nor purified epithelial cells via microdissection prior to RNA extraction. Moreover, Chip-based profiling needs to be validated on a platform with more specificity. Previous studies have relied on established cell lines $(13,21)$, which cannot simulate clinical carcinomas in a perfect manner. Cell lines, for example, accumulate genetic alterations in culture. Similar to other cancers, epithelial ovarian cancer is also a heterogeneous population of cells. In the present study, using LMD, the interference of confounding factors was minimized. The results revealed that the expression of miR-126 was significantly decreased in epithelial ovarian cancer compared with normal ovarian epithelial cells.

VEGF family members, such as VEGF-A, VEGF-B, VEGF-C, VEGF-D, VEGF-E and PGF are essential modulators of angiogenesis. Previous studies have demonstrated that VEGF-A is the direct target of miR-126 in various tumors (22-26). Phase III clinical trials have provided encouraging results for anti-VEGF therapy in ovarian cancer (27). It has been reported that bevacizumab combined with chemotherapy improves progression-free survival and the objective response rate in patients with refractory ovarian cancer (28). Consistent with a previous in vitro study, the results of the present study demonstrated that miR-126 targeted VEGF-A in ES2 and SKOV3 cells and that miR-126 expression negatively correlated with VEGF expression levels in epithelial ovarian cancer tissues. Moreover, the ectopic expression of miR-126 impeded angiogenesis in the tube formation assay. It is important to 

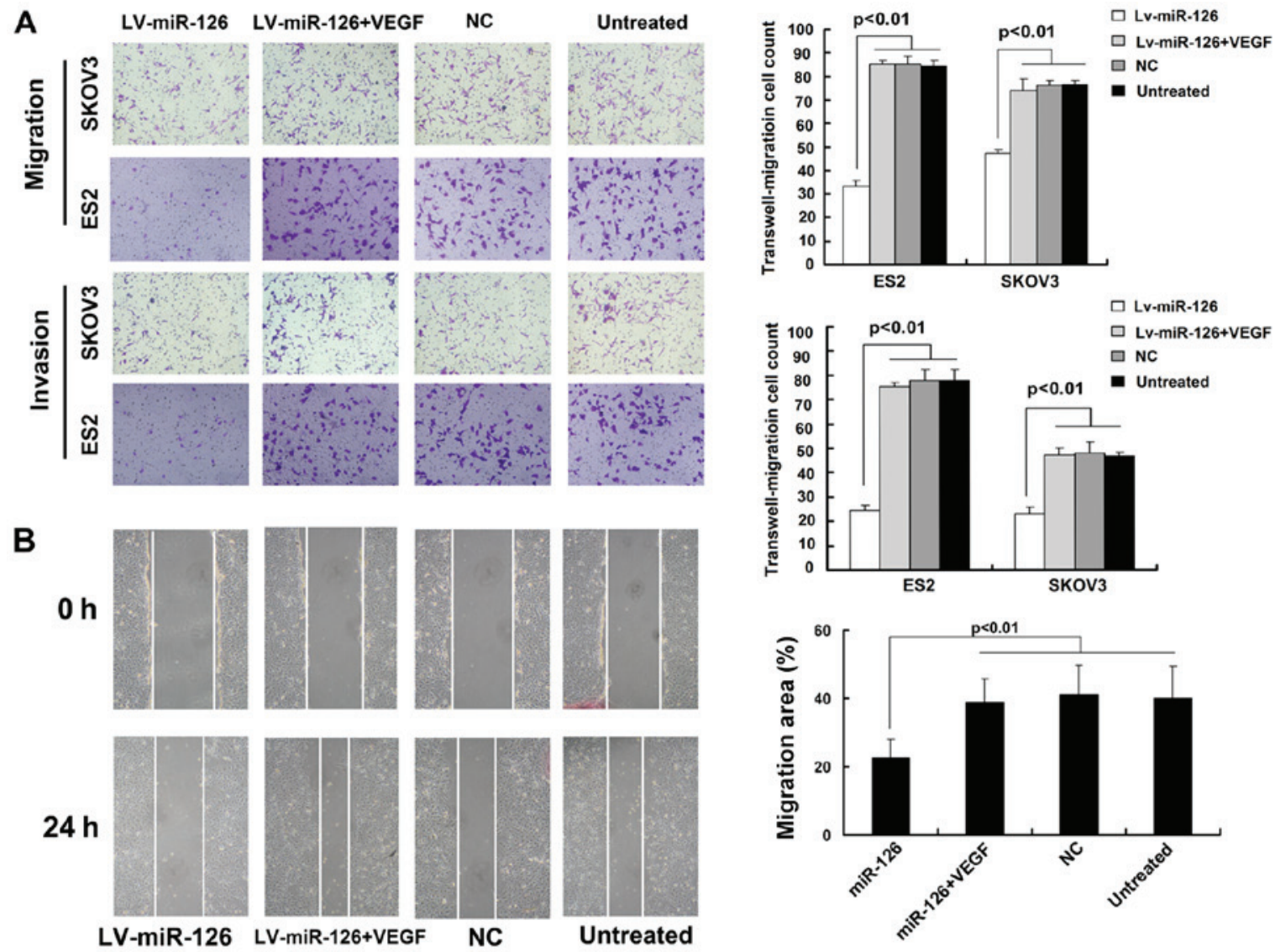

C
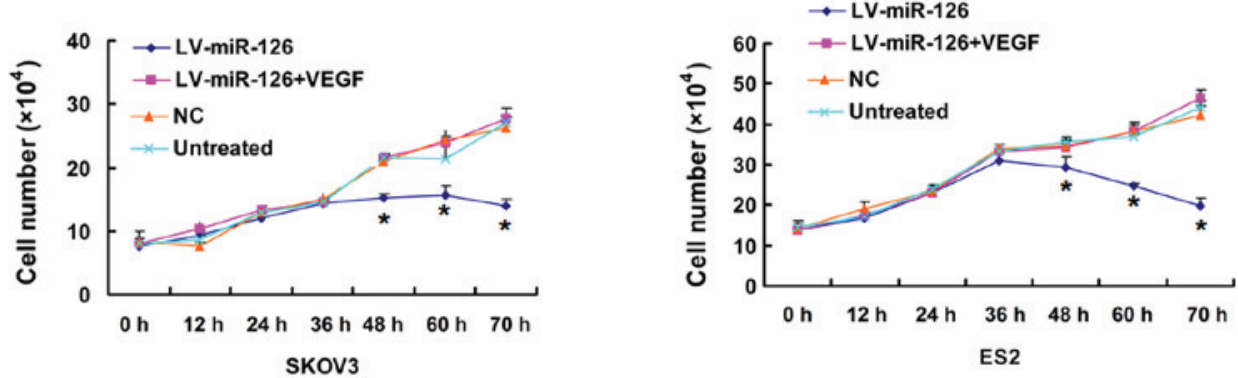

D

$1 \mathrm{~h}$

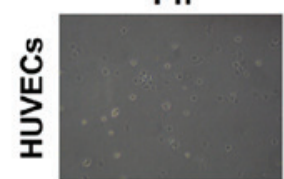

E

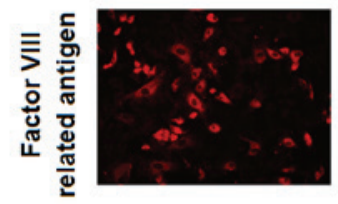

$\mathbf{F}$

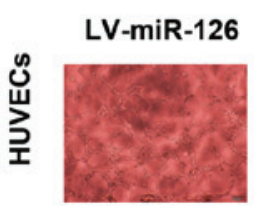

$4 \mathrm{~h}$

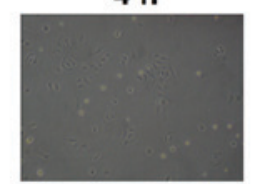

$24 \mathrm{~h}$
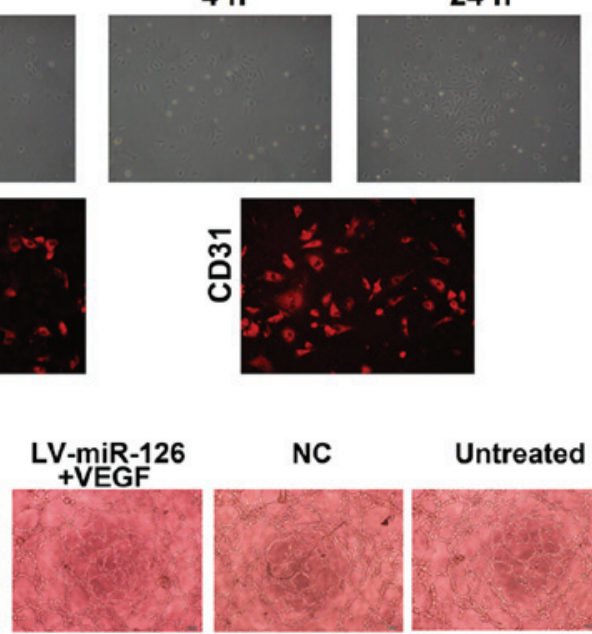

Untreated
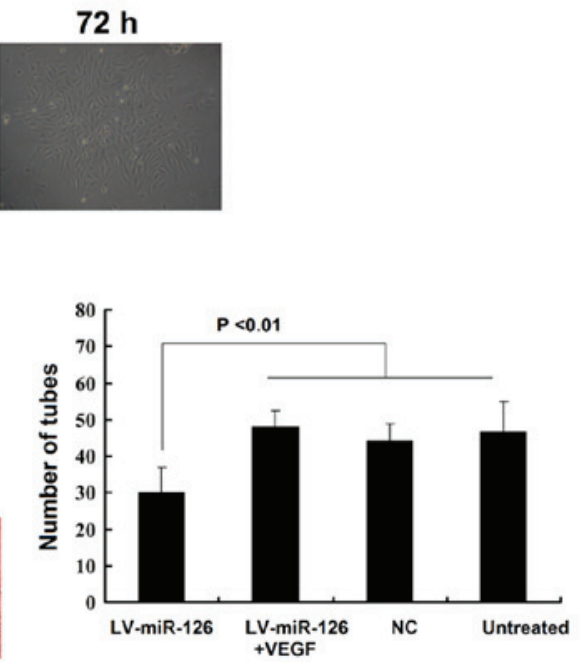

Figure 5. Ectopic expression of VEGF-A attenuates the miR-126-mediated effects on metastasis and proliferation. Recombinant human VEGF was added to lentivirus-miR-126 (LV-miR-126)-infected cells. (A) Transwell migration and invasion assays was used to detect cell proliferation in the LV-miR126, LV-miR-126 + VEGF, NC and untreated groups. (B) Wound-healing assay to measure the migration of the LV-miR126, LV-miR-126+VEGF, NC, untreated cells. (C) Trypan blue dye exclusion assay was conducted to count the number of viable cells at 0,12,24, 36, 48, 60 and $72 \mathrm{~h}$ in SKOV3 and ES2 cells; ${ }^{*} \mathrm{P}<0.05$ compared to untreated cells. (D and E) Identification for isolated HUVECs. (D) These endothelial cells exhibited a polygonal shape and were arranged in a single layer of paving stones under an inverted microscope. (E) Identification for the isolated HUVECs by examining the factor VIII related antigen and CD31. (F) Observation for branching points of dendritic and tube-like structures. NC, negative control. 
A

\section{LV-miR-126}

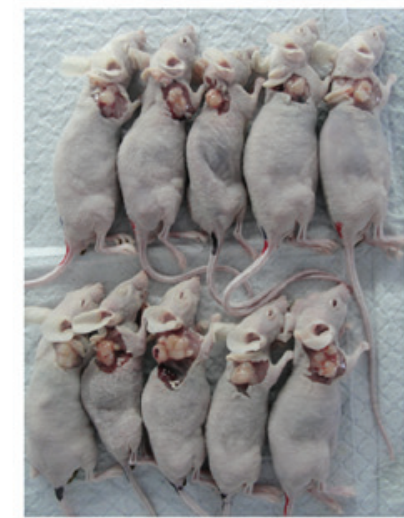

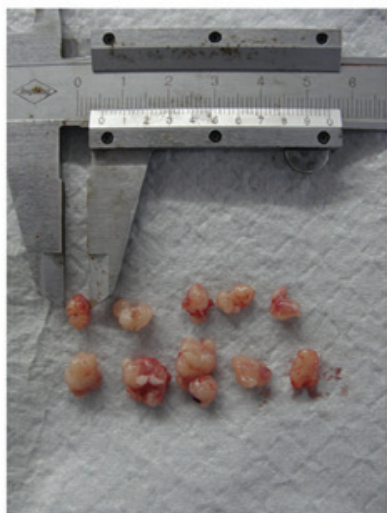

\section{LV-miR-126}

NC
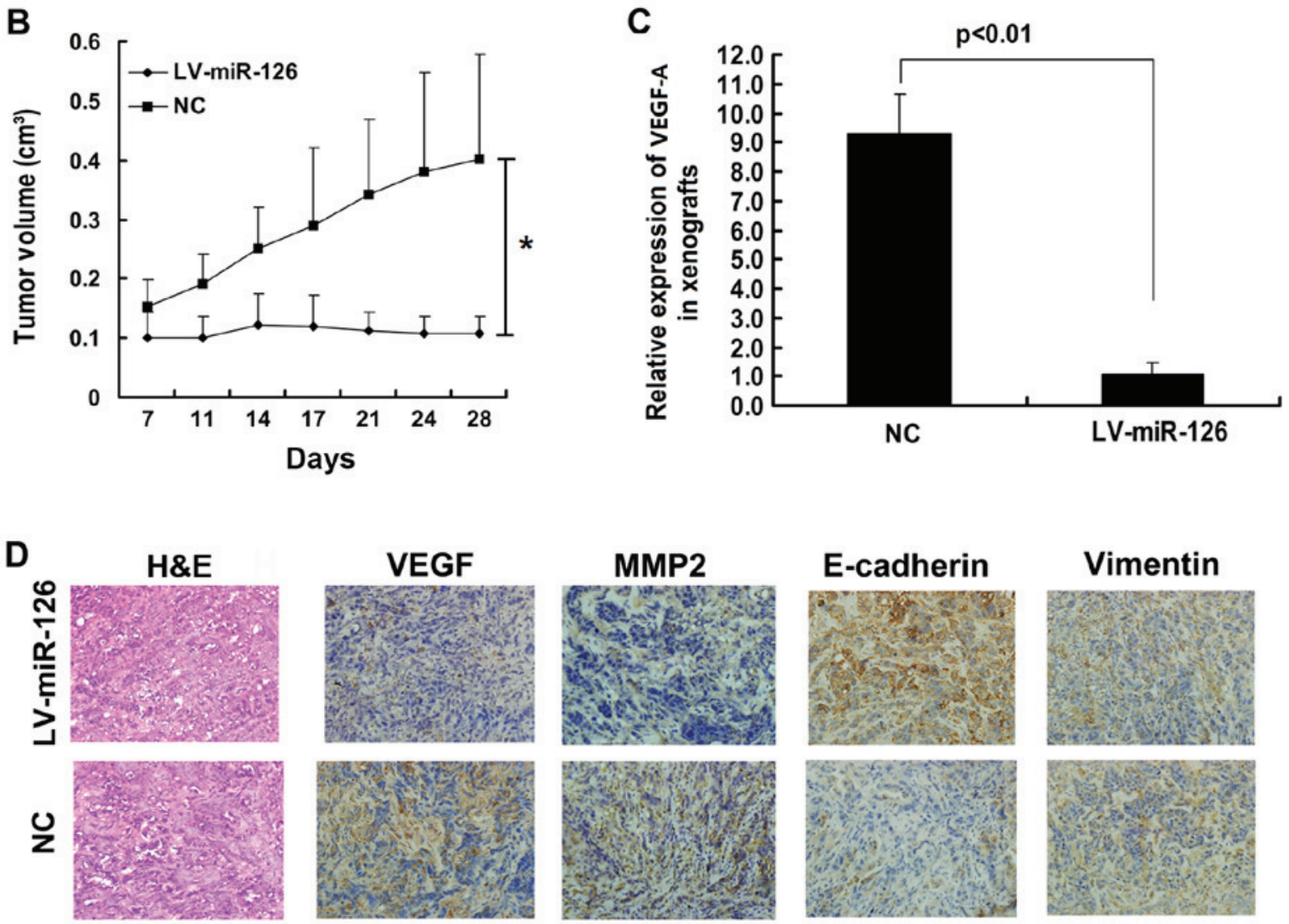

Figure 6. miR-126 inhibits tumor growth in vivo. (A) Representative images of xenograft tumors (n=5) (B) The tumor growth rate of the experimental mice measured by tumor volumes. ${ }^{\mathrm{P}}<0.001$. (C) The mRNA level of VEGF-A in the mouse tumors from the miR-126 overexpression and NC groups. (D) Levels of VEGF-A, MMP2, E-Cadherin and vimentin were determined by immunohistochemistry.

note that the addition of recombinant VEGF-A could not fully mitigate the miR-126-mediated effects, highlighting the pleiotropic effects of miR-126. It is necessary to point out that the present study was correlational and was mainly focused on the association between miR-126 and VEGF-A. There was no direct evidence that miR-126 directly targeted VEGF-A in ovarian cancer and investigating the effects of miR-126 on the other VEGF family members and/or various isoforms of VEGF-A will better reveal the association between miR-126 and VEGF family members. Further studies are thus required.

In conclusion, the present study demonstrated that miR-126 expression was decreased in ovarian cancer and that the decreased expression of miR-126 promoted ovarian cancer angiogenesis and invasion by targeting VEGF-A. Moreover, as metastases and proliferation are responsible for patient mortality in ovarian cancer, the ability of miR-126 to impede angiogenesis and invasion may prove to be clinically useful.

\section{Acknowledgements}

Not applicable.

\section{Funding}

The present study was supported by the National Natural Science Foundation of China (grant no. 81402166). 


\section{Availability of data and materials}

All data generated or analyzed during this study are included in this published article or are available from the corresponding author on reasonable request.

\section{Authors' contributions}

The experiments were designed by JCao and SW. LY and LL performed the experiments (with the assistance of $\mathrm{DH}, \mathrm{QH}$ and JCai) and were responsible for data analysis and the drafting of the article. JCao and SW revised the article critically for intellectual content. All authors have read and approved the final manuscript.

\section{Ethics approval and consent to participate}

Tumor and normal specimens were obtained from the Gynaecologic Tissue Bank of Wuhan Union Hospital with written informed consent and ethical approval. The present study was approved by the ethical committee of Union Hospital, Tongji Medical College, Huazhong University of Science and Technology, China. In addition, human umbilical cords were collected from pregnant women with written informed consent and ethical approval by Ethics Committee of Union Hospital, Tongji Medical College, Huazhong University of Science and Technology, Wuhan, China. All animal experiments were approved by the Institutional Animal Care and Use Committee at Tongji Medical College, Huazhong University of Science and Technology.

\section{Patient consent for publication}

Not applicable.

\section{Competing interests}

The authors declare that they have no competing interests.

\section{References}

1. Siegel RL, Miller KD and Jemal A: Cancer statistics, 2019. CA Cancer J Clin 69: 7-34, 2019.

2. Meister J and Schmidt MHH: miR-126 and miR-126*: New players in cancer. ScientificWorldJournal 10: 2090-2100, 2010.

3. Fish JE, Santoro MM, Morton SU, Yu S, Yeh RF, Wythe JD, Ivey KN, Bruneau BG, Stainier DY and Srivastava D: miR-126 regulates angiogenic signaling and vascular integrity. Dev Cell 15: 272-284, 2008.

4. Zhang Y, Yang P, Sun T, Li D, Xu X, Rui Y, Li C, Chong M, Ibrahim T, Mercatali L, et al: miR-126 and miR-126* repress recruitment of mesenchymal stem cells and inflammatory monocytes to inhibit breast cancer metastasis. Nat Cell Biol 15: 284-294, 2013.

5. Ebrahimi F, Gopalan V, Smith RA and Lam AK: miR-126 in human cancers: Clinical roles and current perspectives. Exp Mol Pathol 96: 98-107, 2014

6. Wang S, Aurora AB, Johnson BA, Qi X, McAnally J, Hill JA, Richardson JA, Bassel-Duby R and Olson EN: The endothelial-specific microRNA miR-126 governs vascular integrity and angiogenesis. Dev Cell 15: 261-271, 2008.

7. Lagos-Quintana M, Rauhut R, Yalcin A, Meyer J, Lendeckel W and Tuschl T: Identification of tissue-specific microRNAs from mouse. Curr Biol 12: 735-739, 2002.
8. Roviello G, Bachelot T, Hudis CA, Curigliano G, Reynolds AR, Petrioli R and Generali D: The role of bevacizumab in solid tumours: A literature based meta-analysis of randomised trials. Eur J Cancer 75: 245-258, 2017.

9. Pan C, Stevic I, Müller V, Ni Q, Oliveira-Ferrer L, Pantel K and Schwarzenbach $\mathrm{H}$ : Exosomal microRNAs as tumor markers in epithelial ovarian cancer. Mol Oncol 12: 1935-1948, 2018.

10. Prahm KP, Høgdall C, Karlsen MA, Christensen IJ, Novotny GW and $\mathrm{H} \varnothing$ gdall E: Identification and validation of potential prognostic and predictive miRNAs of epithelial ovarian cancer. PLoS One 13: e0207319, 2018.

11. Wu G, Cao L, Zhu J, Tan Z, Tang M, Li Z, Hu Y, Yu R, Zhang S, Song L, et al: Loss of RBMS3 confers platinum resistance in epithelial ovarian cancer via activation of miR-126-5p/ $\beta$-catenin/CBP signaling. Clin Cancer Res 25: 1022-1035, 2019.

12. Xiang $G$ and Cheng $Y$ : MiR-126-3p inhibits ovarian cancer proliferation and invasion via targeting PLXNB2. Reprod Biol 18: 218-224, 2018.

13. Luo P, Fei J, Zhou J and Zhang W: microRNA-126 suppresses PAK4 expression in ovarian cancer SKOV3 cells. Oncol Lett 9: 2225-2229, 2015

14. Cai J, Li T, Huang B, Cheng H, Ding H, Dong W, Xiao M, Liu L and Wang Z: The use of laser microdissection in the identification of suitable reference genes for normalization of quantitative real-time PCR in human FFPE epithelial ovarian tissue samples. PLoS One 9: e95974, 2014.

15. Xie H, Zou L, Zhu J and Yang Y: Effects of netrin-1 and netrin-1 knockdown on human umbilical vein endothelial cells and angiogenesis of rat placenta. Placenta 32: 546-553, 2011.

16. Livak KJ and Schmittgen TD: Analysis of relative gene expression data using real-time quantitative PCR and the 2(-Delta Delta C(T)) Method. Methods 25: 402-408, 2001.

17. Li Q, Cheng K, Wang AY, Xu QG, Fu ZF, He SY and Xu PX: microRNA-126 inhibits tube formation of HUVECs by interacting with EGFL7 and down-regulating PI3K/AKT signaling pathway. Biomed Pharmacother 116: 109007, 2019.

18. Guo J, Cai J, Yu L, Tang H, Chen C and Wang Z: EZH2 regulates expression of p57 and contributes to progression of ovarian cancer in vitro and in vivo. Cancer Sci 102: 530-539, 2011.

19. Bu J, Li H, Li XY, Liu LH, Sun W and Xiao T: Prognostic role of microRNA-126 for survival in malignant tumors: A systematic review and meta-analysis. Dis Markers 2015: 739469, 2015.

20. Gu L, Li H, Chen L, Ma X, Gao Y, Li X, Zhang Y, Fan Y and Zhang X: MicroRNAs as prognostic molecular signatures in renal cell carcinoma: A systematic review and meta-analysis. Oncotarget 6: 32545-32560, 2015.

21. Luo J, Zhu C, Wang H, Yu L and Zhou J: MicroRNA-126 affects ovarian cancer cell differentiation and invasion by modulating expression of vascular endothelial growth factor. Oncol Lett 15: 5803-5808, 2018.

22. Chen H, Li L, Wang S, Lei Y, Ge Q, Lv N, Zhou X and Chen C: Reduced miR-126 expression facilitates angiogenesis of gastric cancer through its regulation on VEGF-A. Oncotarget 5: 11873-11885, 2014.

23. Chen CY, Su CM, Hsu CJ, Huang CC, Wang SW, Liu SC, Chen WC, Fuh LJ and Tang CH: CCN1 promotes VEGF production in osteoblasts and induces endothelial progenitor cell angiogenesis by inhibiting miR-126 expression in rheumatoid arthritis. J Bone Miner Res 32: 34-45, 2017.

24. Kong D, Ying B, Zhang J and Ying H: The anti-osteosarcoma property of ailanthone through regulation of miR-126/VEGF-A axis. Artif Cells Nanomed Biotechnol 47: 3913-3919, 2019.

25. Liu B, Peng XC, Zheng XL, Wang J and Qin YW: MiR-126 restoration down-regulate VEGF and inhibit the growth of lung cancer cell lines in vitro and in vivo. Lung Cancer 66: 169-175, 2009.

26. Caporali S, Amaro A, Levati L, Alvino E, Lacal PM, Mastroeni S, Ruffini F, Bonmassar L, Antonini Cappellini GC, Felli N, et al: miR-126-3p down-regulation contributes to dabrafenib acquired resistance in melanoma by up-regulating ADAM9 and VEGF-A. J Exp Clin Cancer Res 38: 272, 2019.

27. Cortez AJ, Tudrej P, Kujawa KA and Lisowska KM: Advances in ovarian cancer therapy. Cancer Chemother Pharmacol 81: 17-38, 2018.

28. Pujade-Lauraine E, Hilpert F, Weber B, Reuss A, Poveda A, Kristensen G, Sorio R, Vergote I, Witteveen P, Bamias A, et al: Bevacizumab combined with chemotherapy for platinum-resistant recurrent ovarian cancer: The AURELIA open-label randomized phase III trial. J Clin Oncol 32: 1302-1308, 2014. 